\title{
Retention of Skills with and without Debriefing Three-Months after Cardiopulmonary Resuscitation Training in Makkah, Saudi Arabia: A Randomized Controlled Trial
}

\author{
Rehab Mohammed Gaafar1*, Soha Aly Elmorsy², Abdulmajeed Soliman Khan ${ }^{3}$, \\ Mohammad Kamel Al-harazi ${ }^{4}$ Shaimaa Kamel Al-harazi ${ }^{5}$, Nouf Kamel Al-harazi ${ }^{6}$ \\ ${ }^{1}$ Saudi Heart Association, Riyadh, KSA \\ ${ }^{2}$ Research Center, King Abdullah Medical City, Makkah, KSA \\ ${ }^{3}$ Internal Medicine Department, Hera General Hospital Makkah, Ministry of Health (MoH), Makkah, KSA \\ ${ }^{4}$ Community Medicine Department, MoH, Hera General Hospital, Makkah, KSA \\ ${ }^{5}$ King Saud University for Health Sciences, Riyadh, KSA \\ ${ }^{6}$ Umm Al-Qura University, Makkah, KSA \\ Email: ^rehab.m.gaafar@gmail.com
}

How to cite this paper: Gaafar, R.M., Elmorsy, S.A., Khan, A.S., Al-harazi, M.K., Al-harazi, S.K. and Al-harazi, N.K. (2019) Retention of Skills with and without Debriefing Three-Months after Cardiopulmonary Resuscitation Training in Makkah, Saudi Arabia: A Randomized Controlled Trial. Open Journal of Preventive Medicine, 9, 141-154.

https://doi.org/10.4236/ojpm.2019.911013

Received: September 8, 2019

Accepted: November 24, 2019

Published: November 27, 2019

Copyright $\odot 2019$ by author(s) and Scientific Research Publishing Inc. This work is licensed under the Creative Commons Attribution International License (CC BY 4.0).

http://creativecommons.org/licenses/by/4.0/

\section{(c) (i) Open Access}

\begin{abstract}
Background: Evidence of cardiopulmonary resuscitation (CPR) performance has driven interest in procedures, e.g., debriefing to improve CPR quality. Aim: To investigate retention of skills with and without debriefing 3-months after CPR training on high-fidelity manikins (HFM) among participants involved in the "Heart Safe City Initiative" event (HSCI) in Makkah, Saudi Arabia. Methodology: A randomized controlled design was used during October 2017-January 2018. Participants were divided into two groups: an intervention group to receive debriefing after CPR (debriefing group or "subjects"), and a non-intervention group ("controls"). Participants were tested repeatedly: before training (pre-training test), immediately after and 3-months after training (retention or late test); and scores for each test were recorded. Results. The study's subjects and controls did not vary by age, sex, nationality, and profession's criteria ( $p>0.05$, all analyses). The mean retention posttests scores significantly varied between subjects and controls $[t(\mathrm{df}=200)=$ 27.7, $p<0.0001)$ ]; however, the two groups did not vary in their immediate score levels (mean ranks: 106.77 v. $95.68, p=0.18$ ). Further, the immediate posttest scores were significantly higher than the pretests' within the study population as a whole group [mean difference $38.05 \% \pm 27.59 \%, t(\mathrm{df}=201)=$ 13.5, $p<0.001$ ]; within subjects [mean difference $40.68 \% \pm 29.26 \%, t(105)=$ $14.31, p<0.0001$ ]; and within controls [mean difference $35.06 \% \pm 25.44 \%$,
\end{abstract}


$t(\mathrm{df}=95)=13.50, p<0.0001]$. The retention scores also behaved likewise [mean difference $40.35 \% \pm 27.5 \%, t(\mathrm{df}=201)=20.85, p<0.0001$; mean difference $52.57 \% \pm 25.22 \%, t(\mathrm{df}=105)=21.46, p<0.0001$; mean difference $26.86 \% \pm 23.39 \%, t(\mathrm{df}=95)=22.12, p<0.0001$, respectively]. Subjects reported a significantly higher retention scores than the immediate scores [mean difference $11.88 \% \pm 25.67 \%, t(\mathrm{df}=200), 4.76, p<0.0001$ ]; compared with controls who performed otherwise (mean difference $-8.19 \% \pm 27.20 \%$, $t(\mathrm{df}=97)=-2.95, p<0.0001)$. Conclusions. Debriefing using HFM training achieves the target of improving the public's skills of CPR. Considering the urgent need to recruit every society member to be a heartsaver; debriefing after CPR training helps improve the quality and retention of basic CPR skills.

\section{Keywords}

Cardiopulmonary Resuscitation, Debriefing, Retention, Skills, Saudi Arabia

\section{Introduction}

Cardiac arrest refers to the sudden cessation of cardiac activity with hemodynamic collapse takes place, typically due to sustained ventricular tachycardia/ventricular fibrillation (VF) [1] [2]. Cardiac arrest strikes suddenly, hence the name "sudden cardiac arrest" (SCA), leaving less than 5\% chance to survive the attack [3]. In addition to the number of lives lost, cardiac arrest has a considerable economic impact; measured in terms of productive years of life lost due to premature death or avoidable neurologic disability, it constitutes a societal burden equal to or greater than that of other leading causes of death in the community [4]. This is why only prevention and the need to develop strategies for management of sudden cardiac death are warranted. Several factors influence the development of risk factors for SCA, including the presence of underlying cardiovascular disease (CVD), including coronary heart disease (CHD), atherosclerosis, hypertension, heart failure, as well as inherited disorders affecting cardiac rhythm, such as long QT interval syndrome [1]. The setting where SCA takes place is an important determinant for the survivability outcome of an SCA incident. Around 347,000 out-of-hospital cardiac arrest (OHCA) and 209,000 in-hospital cardiac arrests (IHCAs) occur in adults each year in the US; millions more occurring across the rest of North America, Europe, and Asia [5]. Cardiac arrest becomes more common with age; and males are more often affected [1]. Sudden cardiac death (SCD) may often follow, unless a successful intervention, e.g., defibrillation or spontaneous reversion restores circulation, has been achieved; in which case the event is referred to as aborted SCD. However, a bradyarrhythmia is responsible for some cases of SCD [2] [6]. Absence of pulse is the prominent sign of cardiac arrest [1], however the exact mechanism of the collapse may be impossible to establish. For the vast majority of patients who die suddenly, cardiac activity is not being monitored at the time of their col- 
lapse. As a result, the mechanism can only be inferred, based upon information obtained after the process has been initiated. On the other hand, there have been many cases in which the initiating event has been witnessed or recorded [6] [7].

Management and prevention through public awareness and preparedness: The prevention of OHCAs is the most important factor for increasing the survival rate. In many cases, time from recognition of cardiac arrest to the arrival of emergency medical service (EMS) is long, leaving bystanders in a critical position to potentially influence patient prognosis. Prior training in CPR and use of AED may influence bystander interventions. The European Resuscitation Council has published that there will be a European cardiac arrest awareness day each year (Oct. 16) [8]. More than 70 percent SCA incidents occur in the home, which is why home AEDs have the potential to save the lives of countless loved ones struck by cardiac arrest.

The sequence of actions whereas joining the victim of SCA with survival is called "chain of survival (COS)" [9]. System-based improvements to the COS had yielded increases in survival from OHCA. However, in order for the patient to have the best chance of surviving an OHCA, early recognition, early CPR and defibrillation must be provided within the first 4 minutes of the cardiac arrest. Geri et al. [10] define CPR as a therapeutic intervention performed for patients with cardiac and respiratory arrest. Bystander CPR is a key life-saving factor in the COS. Further, adequate post-resuscitation care of OHCA victims should be considered for transportation to a specialized cardiac arrest center as part of a broader regional system of care [9]. The Saudi Heart Association (SHA) set CPR guidelines practically suite SCA and chocking incidents in the Saudi populations [9]. The SHA recommends starting CPR with the sequence of circulation-airway-breathing $(\mathrm{CAB})$ in the pre-hospital situations, while the sequence of $A B C$ is still effective within the healthcare facilities. Thereby, delivering proper starting chest compressions as soon as possible before trying to open the airway and give rescue breathing is vital to high-quality CPR and optimizes the chance of return of spontaneous circulation and surviving the attack [11]. Unfortunately, the lack of interest in learning CPR and the fear of doing something wrong or of legal liability are obstacles limiting bystander to learn and perform CPR. Further, the retention of skills after CPR training has been demonstrated to be poor in several domains after only a few months [12].

Debriefing and retention of resuscitation performance: Debriefing involves a guided reflection in the cycle of experiential learning [13]. Especially in medical emergency, debriefing is a powerful tool to assure retention of resuscitation practice knowledge and skills, and hence positively influence patient outcome [14]. A variety of debriefing approaches are available to help refresh and maintain previously acquired CPR skills [15] [16]. Pre-training evaluation and feedback have been shown to improve CPR skills immediately following training. The need for efficient retraining is obvious, but the optimal format for refresher training is still one of the knowledge gaps to be addressed [14]. The research 
suggests that resuscitation training should be carried out at least every three to six months to prevent decay of information and skills which may well be lost during this duration. A resuscitation manikin with a real-time feedback mechanism to allow self-study and practice enhances performance both during training and after debriefing [15] [16]. New HFM designed for timely measuring of the core components of CPR performance (e.g., compression rate and depth, ventilation volume), using intuitive graphics and easy-to-follow guidance have been in line [17] [18].

Creating communities of lifesavers. After one minute of cardiac arrest, brain cells without oxygen begin to die. Meanwhile, an EMS response time can average 7 - 12 minutes, or even longer. By increasing bystander CPR works, there is a huge potential for increasing survival rates. The HSCI of Makkah [19] is a public safety program sponsored by Makkah governorate in partnership with the Ministry of Health $(\mathrm{MoH})$ and Ministry of Education $(\mathrm{MoE})$ in collaboration with SHA. The program seeks to save emergency heart conditions and proceed directly to the nearest health facility in a time consistent with what is universally recognized. Given the importance of Makkah in receiving huge numbers of pilgrims annually, improving the outcomes of OHCAs through training of target groups, civil defense staff, scouts, and hotels personnel around Makkah holy places is mandated. In addition, providing AEDs at all these places and equipping mobile teams with such devices, especially during peak times in the central Makkah region would be so helpful.

Rationale and study aim: One of the essential skills to achieve the prospective national health goals is having qualified public performing excellent life-saving $\mathrm{CPR}$ which is a cornerstone for life-saving following OHCA. Studies on the role of retention of the public's CPR skills through debriefing in KSA are scarce. To the best of our knowledge, no studies were conducted concerning the CPR skills retention among public living in Makkah. The aim of the current study was to investigate retention of skills with and without debriefing three months after CPR training on HFMs among participants involved in the HSCI program of Makkah during the period from October 2017-January 2018. The participants' ability to recall their performance, their response to debriefing after immediate post-training as a criterion standard, would be evaluated.

\section{Subjects and Methods}

A randomized controlled approach was adopted to achieve study aim. The experiment was held at the Simulation Center at Hera General Hospital, Makkah, KSA. Knowingly, Makkah is the holiest city to all Muslims and the spiritual capital of KSA. The study population encompassed all participants targeted in the HSCI program of Makkah during the period from October 2017 until January 2018. All male and female participants of all nationalities and professions would be included in the study, and only those who refused to participate were excluded.

Study sampling. The sample size for this study was based on primary out- 
come variable which was the score of retention 3-month post-training test for two groups of participants: experimental group (those who would receive debriefing among the training plan, and those who would not, i.e., control group) (see randomization). The score was calculated as a percentage out of 100 . The sample size $(n)$ was generated using the formula: $n=f(\alpha, \beta) * 2 \alpha^{2} /\left(\mu_{1}-\mu_{2}\right)^{2}$ (where: $\mu_{1}=$ mean test score in the debriefing group, $\mu_{2}=$ mean test score in the control group, $\sigma=$ standard deviation (SD) of 3-month post-training score, mean difference in the same score between experimental group and control group and $=30$, assumptions of " $n$ " $=10$, SD of the retention post-training score $=10, \alpha=0.05$, power $=80 \%)$ (http://www.sample-size.net/sample-size-proportions/). A sample size of 84 per group was calculated. In order to account for withdrawals, loss to follow up (LFU), or invalid data, " $n$ " would be increased to 100 per group. Participants were stratified by gender and then from each stratum an equal number of participants was selected using a simple random technique (using a true random number generator) (http://www.random.org/).

Data collection: A registration form predesigned by the HSCI program was used to record the participants' demographic data, including name, ID, date of birth, address, qualifications, occupation and working place. The training was on HFMs which were set up according to the standard CPR requirements of the SHA (connected to SimPad PLUS with SkillReporter to record data) [9]. Participants were asked to respond to a case scenario and were applying their skills on the HFM. Each participant was required to give constructive feedback while performing the CPR. The summary screen provides instant feedback of the session to debrief participant individually, (up to six manikins could be controlled while using SimPad PLUS with SKillReporter at a time). The training sessions were held on Sunday and Monday of every week for 16 weeks of study duration. Participants were briefed of the study's aim and procedures; a verbal consent was considered as an acceptance to participate. The participants were further reassured of the voluntary nature of the study and that they could opt out of it at any time without giving reasons. They were also assured of the utmost confidentiality of the collected information and that only grouped and unanimous data would be disclosed for scientific purposes. At the beginning of the training day, participants would check in and fill the registration form. The session starts by pre-training test, and then to watch a video for an hour (the video demonstrates standard CPR for adults, children, and infants, and AED and choking rescue). Presentation then wraps up by answering participants' questions.

Randomization: By the end of training session, each participant chose between two papers: one for debriefing and the other for control. Learners who choose the debriefing paper would be given a feedback on their performance and allowed to reflect by the instructor. Those who chose the control group, the instructor greeted them and asked them to come back again after three months for the post-training test. Following the trial, the instructor recorded the performance of a one-minute compression and ventilation-only CPR. By using SimPad PLUS with SkillReporter, each trainee performance was recorded by his/her 
name and compared with his/her retention test score. The passing score on each test was $50 \%$. The score of final result includes the total mark of compression rate and depth and ventilation volume which was in total out of $100 \%$. A participant who scores less than $50 \%$ would be debriefed again and the immediate post-training test score retaken.

Study variables and statistical analyses. The independent study variables include demographic data, as above, intervention (debriefing), pre-training test scores (often called pretest score), and immediate post-training score. The study's dependent variable is represented by retention 3-month post-training score (often called retention or late test). First, descriptive statistics, including frequency data, would be displayed. Qualitative data, e.g., sex, profession, were summarized as count (\%), and quantitative data, e.g., age and scores were summarized as the mean $\pm \mathrm{SD}$, range or the median [interquartile range (IQR)], where appropriate. The study's inferential statistics plan was set so that first, the difference in scores of the selected tests would be analyzed between subjects and controls, e.g. to measure the influence of training upon the subjects' retained CPR skills; second, the difference in scores would be measured within each individual group, e.g., debriefing group or the control group, and also within the study population as a whole group, and so forth. Utilized statistical techniques included parametric techniques (PMTs), such as $t$-test, e.g., to measure the difference between the means scores of two groups. In which case normality assumption would first be assessed suing normality tests, such as Shapiro Wilk test. Otherwise, a non-PMT alternative test, such as Mann-Whitney $U$, would be used. Likewise, paired $t$-test sample for the difference in the mean scores, e.g., before and after debriefing may be used. Chi-square test, or Fisher's exact, where appropriate, would be used to test the differences between two categorical groups. Pearson correlation was conducted to assess the linear relationship between the retention score and other continuous variables, such as age, pretest score, and immediate score. The SPSS for Windows, version 21 (IBM Corp. Armonk, NY, USA) was used to perform the study's statistical analyses. Our alpha level for tolerating type- 1 error was $\alpha=0.05$; results with $p$-value $<0.05$ were considered significant. An approval to conduct the study was obtained from the concerned agencies, including the Research Ethics Committee of the General Directory of Health Affairs of Makkah, MoH. A permission to conduct the experiment at Hera General Hospital was also granted from the hospital's administration.

\section{Results}

Among a total 202 participants, $106(52.5 \%)$ were men and $96(47.5 \%)$ were women, with mean age $37.62 \pm 9.40$ (Table 1). Only 11 (5.4\%) were non-Saudi and 191 (94.6\%) were Saudi. The number of participants with teaching profession accounted $78(81.3 \%)$ in the debriefing group and $68(70.8 \%)$ in the control group, while 23 (11.4\%) participants were staff from Umm Al-Qura University (UQU) (in the two groups). Otherwise, neighborhood-centers participants were 
males with different age strata 29 (14.4\%); and students were only $4(4.2 \%)$ in both groups (Table 1).

Only whole study population's retention score was significantly higher among debriefing than the control group [means $=65.2 \% \pm 16.8 \%$ and $32.4 \pm 18.2 \%$, respectively, $t(\mathrm{df}=200)=27.7, p<0.001]$ (Table 2). There were not significant differences in the two group's scores either before or immediately after training ( $U=5079.5, p=0.98 ; U=4529.5, p=0.18$, respectively).

As in Table 3, individual "within group" comparisons are conducted. The immediate scores were significantly higher than pretest scores in the three analyses: whole study population [mean difference $38.05 \% \pm 27.59 \%, t(\mathrm{df}=201)=$ $13.5, p<0.001$ ]; debriefing group [mean difference $40.68 \pm 29.26, t(\mathrm{df}=105)=$ $14.31, p<0.0001$ ]; and control group [mean difference $35.06 \% \pm 25.44 \%, t(\mathrm{df}=$ $95)=p<0.0001]$. Likewise, the retention scores were significantly higher than those before the training for all analyses: all study population $(\mathrm{n}=202)$ [mean difference $40.35 \% \pm 27.5 \%, t(201)=20.85, p<0.0001]$; debriefing group $(\mathrm{n}=$ 106) [mean difference $52.57 \% \pm 25.22 \%, t(105)=21.46, p<0.0001$ ]; control group $(\mathrm{n}=96)$ [mean difference $26.86 \% \pm 23.39 \%, t(95)=22.12, p<0.0001$ ]

In Table 4, the debriefing group reported significantly higher scores in 3 -month than the immediate post-training scores [mean difference $11.88 \% \pm$ $25.67 \%, p<0.0001]$. Conversely, controls had performed significantly worse on the 3-month test than the immediate test (mean difference $-8.19 \% \pm 27.20 \%, p<$ $0.0001)$.

In Table 5, there was a significantly mild correlation between the retention and immediate post-training scores of the study population as a whole group ( $\mathrm{r}$ $=0.37, p<0.0001)$. The debriefing group scored significantly higher on the retention test than the controls $[58.84 \% \pm 20.89 \%$ vs. $33.60 \% \pm 19.01 \%, t(\mathrm{df} 200)=$ $8.76, p<0.0001]$. Teachers also tended to score higher on the same test than other professions $[49.81 \pm 22.28$ vs. $39.13 \% \pm 26.61 \%, t(\mathrm{df} 200)=2.88, p<$ $0.004]$.

Table 1. Comparing intervention and control groups by demographics criteria.

\begin{tabular}{cccc}
\hline Characteristics & $\begin{array}{c}\text { Debriefing group } \\
(\mathrm{n}=106)\end{array}$ & $\begin{array}{c}\text { Control group } \\
(\mathrm{n}=96)\end{array}$ & Total \\
\hline $\begin{array}{c}\text { Age }(\mathrm{y}) \\
\text { Mean } \pm \text { SD } \\
\text { Sex }\end{array}$ & $38.31 \pm 8.63$ & $36.82 \pm 10.12$ & $37.62 \pm 9.4$ \\
Male & $58(54.7)$ & $48(45.3)$ & $106(100.0)$ \\
Female & $48(50.0)$ & $48(50.0)$ & $96(100.0)$ \\
Nationality & $100(52.3)$ & $91(47.7)$ & $191(100.0)$ \\
Saudi & $6(54.5)$ & $5(45.5)$ & $11(100.0)$ \\
Non-Saudi & $78(53.4)$ & $68(46.6)$ & $146(100.0)$ \\
Specialty & $14(60.9)$ & $9(39.1)$ & $23(100.0)$ \\
Teachers & $4(100)$ & - & $4(100.0)$ \\
UQU staff & $10(34.5)$ & $19(65.5)$ & $29(100.0)$ \\
Students & & & \\
Neighborhood centers & & & \\
\hline
\end{tabular}


Table 2. Pretest, immediate, and 3-month retention scores: Debriefing group vs. control group comparisons [n (debriefing) $=106][\mathrm{n}($ control $)=96]$.

\begin{tabular}{ccccc}
\hline Score category & $\begin{array}{c}\text { Debriefing group } \\
(\mathrm{n}=106)\end{array}$ & $\begin{array}{c}\text { Control group } \\
(\mathrm{n}=96)\end{array}$ & Test statistic & $p$-value \\
\hline $\begin{array}{c}\text { Pre-training } \\
\text { Mean rank }\end{array}$ & 101.42 & 101.59 & $U=5079.5$ & 0.98 \\
$\begin{array}{c}\text { Immediate post-training** } \\
\text { Mean rank }\end{array}$ & 106.77 & 95.68 & $U=4529.5$ & 0.18 \\
$\begin{array}{c}\text { Retention post-training } \\
\text { Mean } \pm \text { SD (\%) }\end{array}$ & $65.2 \pm 16.8$ & $32.4 \pm 18.2$ & $t(\mathrm{df}=200)=27.7$ & $<0.001$ \\
\hline
\end{tabular}

* Debriefing: Mean: $6.7 \pm 14.0$; median 3.5; Controls: Mean $6.7 \pm 14.0$, median 3.7. ${ }^{* *}$ Debriefing: Mean: $49.8 \pm 23.1$; median 50.0; Controls: Mean $46.6 \pm 24.1$, median 50.5.

Table 3. Pre-training and post-training scores: Within group comparisons.

\begin{tabular}{ccccc}
\hline Score category & $\begin{array}{c}\text { Mean } \\
\text { difference }(\%)\end{array}$ & $\begin{array}{c}\text { SD } \\
(\%)\end{array}$ & Test statistic & $p$-value \\
\hline Pre-training-and immediate post-training & & & & \\
All study population score $(\mathrm{n}=202)$ & 38.05 & 27.59 & $t(\mathrm{df} 201)=19.58$ & $<0.0001$ \\
Debriefing group score $(\mathrm{n}=106)$ & 40.68 & 29.26 & $t(\mathrm{df105})=14.31$ & $<0.0001$ \\
Control group scores $(\mathrm{n}=96)$ & 35.06 & 25.44 & $t(\mathrm{df95})=13.50$ & $<0.0001$ \\
Pre-training and retention post-training & & & & \\
All study population score $(\mathrm{n}=202)$ & 40.35 & 27.50 & 20.85 & $<0.0001$ \\
Debriefing group score $(\mathrm{n}=106)$ & 52.57 & 25.22 & 21.46 & $<0.0001$ \\
Control group score $(\mathrm{n}=96)$ & 26.86 & 23.39 & 22.12 & $<0.0001$ \\
\hline
\end{tabular}

Table 4. Comparison of immediate and 3-months post-training-retention scores.

\begin{tabular}{ccccc}
\hline $\begin{array}{c}\text { Immediate and 3-months } \\
\text { post-training }\end{array}$ & $\begin{array}{c}\text { Mean difference } \\
(\%)\end{array}$ & $\begin{array}{c}\text { SD } \\
(\%)\end{array}$ & Test statistic & $p$-value \\
\hline All study population $(\mathrm{n}=202)$ & 2.34 & 28.19 & $t(\mathrm{df} 200)=1.18$ & 0.239 \\
Debriefing group $(\mathrm{n}=106)$ & 11.88 & 25.67 & $t(\mathrm{df} 200)=4.76$ & $<0.0001$ \\
Control group $(\mathrm{n}=96)$ & -8.19 & 27.20 & $t(\mathrm{df} 97)=-2.95$ & $<0.0001$ \\
\hline
\end{tabular}

Table 5. Factors affecting 3-months post-training retention scores of the study population.

\begin{tabular}{cccc}
\hline Factor & Mean score \pm SD (\%) & Test statistic & $p$-value \\
\hline Age & $37.62 \pm 62$ & $r=0.05$ & 0.442 \\
Pre-training score & $6.49 \pm 13.22$ & $r=-(0.01)$ & 0.880 \\
Immediate post-training score & $44.50 \pm 26.38$ & $r=0.37$ & $<0.0001$ \\
Gender & Male: $48.19 \pm 23.55$ & $t=1.28$ & 0.200 \\
& Female: $44.57 \pm 24.36$ & & \\
Debriefing & With: $58.84 \pm 20.89$ & $t=8.76$ & $<0.0001$ \\
& Without: $33.60 \pm 19.01$ & & \\
Being a teacher & Yes: $49.81 \pm 22.28$ & $t=2.88$ & $<0.004$ \\
& No: $39.13 \pm 26.61$ & & \\
Nationality & Saudi: $46.28 \pm 23.80$ & $t=1.40$ & 0.160 \\
& Non-Saudi: $56.73 \pm 26.00$ & & \\
\hline
\end{tabular}




\section{Discussion}

Cardiac arrest is a universal health problem correlated with high levels of mortality [3]. During the VF dysrhythmia which usually takes place in OHCA [2], CPR does provide a source of oxygenation to the tissues and may lead to restoration of a viable cardiac rhythm [20] [21]. The earlier and more successful CPR, the more likelihood of a favorable cardiac arrest outcome [9]. Since most OHCA occurs in the home and public places, training public about quality CPR has the potential to save the lives of countless loved ones stricken by cardiac arrest. Alarmingly, too, OHCAs may often originate from non-cardiac conditions, e.g., trauma, drowning, drug overdose, electrocution [22] [23]. Yet, most OHCA incidents, irrespective of the etiology, do not get bystander-assisted CPR [24]. The American College of Emergency Physicians (ACEP) supports and strongly encourages CPR training for the lay public. This training should be short, easy to understand, and easy to remember [25]. This study aimed to investigate retention of skills with and without debriefing 3 months after CPR training among participants involved in HSCI program. Most of the participants were teachers, probably because the study's data collection coincided with the MoE-sponsored HSCI program, the enrollees of which are MoE-affiliated staff and school teachers.

The current study demonstrates that people debriefed about their CPR performance could achieve a higher score on 3-month post-training test compared with those who were not. This finding is consistent with some other studies [26] [27], with some differences in study design and the outcome measures used. First, our participants were randomly assigned to the study tracks and their resuscitation skills were evaluated with the same system. Second, we took the time needed for debriefing among intervention group and assessed appropriate high-quality CPR skills. Previous studies [26] [27] only used a questionnaire to evaluate knowledge. In support of our finding, too is that multiple guidelines recommend debriefing of resuscitations to improve retention of CPR skills [15]. Fanning et al. (2000) [28] found that much of the research regarding teaching adults indicates that active "participation" is an essential factor in increasing the effectiveness of learning and retained knowledge in this population. Although many learning practices require feedback, debriefing is a particular kind of feedback process. On the other hand, there is substantial evidence about the declination of CPR skills after the initial training [15]. However, there was not a consensus regarding the optimal time interval between practice and the evaluation results at the end of CPR training [27]. Moser et al. studied CPR skills retention in 31 family members of cardiac patients. Of those tested at seven months, more than half were rated "poor" in the initial assessment, chest compression, ventilation, and overall CPR [29]. Thiagarajan et al. [30] reported that, although many learning practices require feedback to keep in mind, debriefing was the best feedback process to enhance improvement of learning. Basic skills, such as asking for help; chest compression, and ventilation, decay 3 - 6 months 
after the training. However, these data were based on independent studies regarding the courses' duration and design, characteristics of instructors and participants and frequency of participants' involvement in real resuscitations [27] [31]. Woollard et al. [32] training laymen from a UK airport, observed that the interval in CPR training should not exceed 7 months. Riegel et al. [29] studying lay volunteers, found a satisfying degree of retention in CPR and AED, even after as long as 17 months of primary training.

In a telephone survey about public knowledge and attitudes towards CPR in Hong Kong [26], the skills appeared to have deteriorated with time. The present study expectedly shows a deficient quality of CPR skills regarding pre-training test performance among all participant groups. This result is similar to Edelson et al. [33] who showed that CPR performance immediately after training was as limited as good as that seen with basic Heart saver training. Korttila et al. [34] reported that CPR performance of the laymen was measured with a recording manikin before 2-months after the training. Before training, none of the subjects passed the surprise resuscitation test. Fernandes et al. [27] reported that $96 \%$ of their high-school students reported meager rates of correct answers before training about essential life support. The present study showed significant high rates of the immediately posttest scores both in the debriefing and the control groups. As in this work, the use of standardized training kits, including videos and manikins, proved effective in resuscitation training. Ribeiro et al. [35] demonstrated the effectiveness of a commercially available training tool encompassing a video and manikin in immediate and delayed retention of knowledge and skills 6 months after CPR training. Using debriefing as the principal intervention to measure its effect on retaining previously-acquired CPR skills in this study allowed our participants to reflect on their performance regarding their psychomotor skills immediately after training and highlight where they performed well and where they needed to improve. Such immediate post-event debriefing has been shown to yield significant results regarding improving performance when combined with hands-on training [36] [37]. The HFM proved a valuable tool, since it promotes physiological changes via an electronic system in response to training, and registers performance in a timely fashion [36]. High-fidelity-based simulation enables a lay bystander to experience such scenarios and potentially reduces anxiety in real situations [38]. In line with the notion that high-quality CPR by far requires building-up efficacious psychomotor skills of trainee candidates, the current study focused primarily on practice regarding measuring skills in performance throughout the training experience, thanks to providing hands-on training and debriefing to the study subjects. With this respect, the study shows a high rate of CPR skills retention 3-months after training in the intervention group. Miotto et al. (2010) [38], and Everett-Thomas et al. (2016) [39], too provide evidence to the positive role of hands-on training combined with debriefing upon CPR performance. In contradiction, theoretical training alone has been shown to produce poor-quality gains [38] [39]. 
Our study presents that being a teacher, immediate post-training test status, and debriefing are likely predictors for a positive change in the retention test performance, a finding that pretty much agrees with what has been found in literature [36] [37]. However, age, gender and nationality did not influence the retention score, e.g., in disagreement with Papalexopoulou et al. [40] who reported that age, and education could affect retention of CPR/AED skills in lay rescuers. As age progresses, one may become more and more unfamiliar with computer-based presentations, as well as with the use of technical equipment, such as the manikins or the AED [40]. The study may have been limited by the inability to recruit a wide scope of Makkah population. However, being the first randomized controlled study to compare retention of CPR skills among the general public of Makkah adds to the experimental design adopted, in addition to the blindness of participants to the performance measurements used. Based on the findings of the current study, it can be concluded that retention of CPR skills among Makkah people was improved with debriefing compared with non-debriefing. Debriefing does enhance retention in the publics' group for 3-months after the initial training. High-quality CPR and the percentage of compressions at correct depth, all are improved, significantly. As such, we recommend encouraging the public to attend CPR courses, pretty much of which are sponsored by the SHA in collaboration with $\mathrm{MoH}$. Participant would rather be debriefed after the CPR session to enhance the retention of skills. Putting in place refresher courses, especially for those types of participants would also be useful in achieving the ultimate goal of turning Makkah into a heart safe city.

\section{Acknowledgements}

We wish to express our sincerest thanks to Dr. Bakr Kalo for his helpful advice and unlimited assistance. We also thank Dr. Noha Saleh Mohamed, professor of public health, High Institute of Public Health; Alexandria University, Egypt for her valuable advice on the methodological and statistical plan outlines for this work. Also, thanks to all the members and participants of "A Heart Safe City Initiative" for their cooperation and kindness. Many thanks to Miss. Faizah Al-Dosary and Mr. Maher Al-Hazmi for their scientific and technical support.

\section{Conflicts of Interest}

The authors declare no conflicts of interest regarding the publication of this paper.

\section{References}

[1] National Heart, Blood, and Long Institute (2016) What Causes Sudden Cardiac Arrest? Washington DC.

https://web.archive.org/web/20160728042233/http://www.nhlbi.nih.gov/health/heal th-topics/topics/scda/causes

[2] Zinckernagel, L., Hansen, C.M., Rod, M.H., Folke, F., Torp-Pedersen, C. and Tjørnhøj-Thomsen, T. (2016) What Are the Barriers to Implementation of Cardi- 
opulmonary Resuscitation Training in Secondary Schools? A Qualitative Study. BMJ Open, 6, e010481. https://doi.org/10.1136/bmjopen-2015-010481

[3] Mehra, R. (2007) Global Public Health Problem of Sudden Cardiac Death. Journal of Electrocardiology, 40, S118-S122. https://doi.org/10.1016/j.jelectrocard.2007.06.023

[4] Stecker, E.C., Reinier, K., Marijon, E., Narayanan, K., Teodorescu, C., Uy-Evanado, A., Gunson, K., Jui, J. and Chugh, S.S. (2014) Public Health Burden of Sudden Cardiac Death in the United States. Arrhythmia \& Electrophysiology, 7, 212-217. https://doi.org/10.1161/CIRCEP.113.001034

[5] Georgiou, M. and Lockey, A.S. (2013) The European Cardiac Arrest Awareness Day. Best Practice \& Research Clinical Anaesthesiology. Arrhythmia \& Electrophysiology, 27, 307-315. https://doi.org/10.1016/j.bpa.2013.07.004

[6] Dubner, S.J., Pinski, S., Palma, S., Elencwajg, B. and Tronge, J.E. (1989) Ambulatory Electrocardiographic Findings in Out-of-Hospital Cardiac Arrest Secondary to Coronary Artery Disease. American Journal of Cardiology, 64, 801. https://doi.org/10.1016/0002-9149(89)90769-8

[7] Bayés de Luna, A., Coumel, P. and Leclercq, J.F. (1989) Ambulatory Sudden Cardiac Death: Mechanisms of Production of Fatal Arrhythmia on the Basis of Data from 157 Cases. American Heart Journal, 117, 151-159. https://doi.org/10.1016/0002-8703(89)90670-4

[8] Hwang, S.O., Chung, S.P., Song, K.J., Kim, H., Rho, T.H., Park, K.N., Kim, Y.M., et al. (2016) Part 1. The Update Process and Highlights: 2015 Korean Guidelines for Cardiopulmonary Resuscitation. Clinical and Experimental Emergency Medicine, 3, S1-S9. https://doi.org/10.15441/ceem.16.133

[9] Saudi Heart Association, National CPR Committee (2015) The Evidenced-Based 2015 CPR Guidelines. Riyadh. http://ksacpr.org.sa:8080/GSSHYD-DT5381/UploadData/CourseContent/b4c41951 -f983-4ba6-ae68-81779364acb0.pdf

[10] Geri, G., Fahrenbruch, C., Meischke, H., Painter, I., White, L., Rea, T.D., et al. (2017) Effects of Bystander CPR Following Out-of-Hospital Cardiac Arrest on Hospital Costs and Long-Term Survival. Resuscitation, 115, 129-134. https://doi.org/10.1016/j.resuscitation.2017.04.016

[11] Meaney, P.A., Bobrow, B.J., Mancini, M.E., Christenson, J., de Caen, A.R., Bhanji, F., et al. (2013) Cardiopulmonary Resuscitation Quality: [Corrected] Improving Cardiac Resuscitation Outcomes Both inside and outside the Hospital: A Consensus Statement from the American Heart Association. Circulation, 128, 417-435. https://doi.org/10.1161/CIR.0b013e31829d8654

[12] Boet, S., Borges, B.C., Naik, V.N., Siu, L.W., Riem, N., Chandra, D., Bould, M.D. and Joo, H.S. (2011) Complex Procedural Skills Are Retained for a Minimum of $1 \mathrm{yr}$ after a Single High-Fidelity Simulation Training Session. British Journal of Anaesthesia, 107, 533-539. https://doi.org/10.1093/bja/aer160

[13] Kessler, D.O., Cheng, A. and Mullan, P.C. (2015) Debriefing in the Emergency Department after Clinical Events: A Practical Guide. Annals of Emergency Medicine, 65, 690-698. https://doi.org/10.1016/j.annemergmed.2014.10.019

[14] Couper, K. and Perkins, G.D. (2013) Debriefing after Resuscitation. Current Opinion in Critical Care, 19, 188-194. https://doi.org/10.1097/MCC.0b013e32835f58aa

[15] Tannenbaum, S.I. and Cerasoli, C.P. (2013) Do Team and Individual Debriefs Enhance Performance? A Meta-Analysis. Human Factors, 55, 231-245. https://doi.org/10.1177/0018720812448394 
[16] De Vries, W. and Handley, A.J. (2007) A Web-Based Micro-Simulation Program for Self-Learning BLS Skills and the Use of an AED. Can Laypeople Train Themselves without a Manikin? Resuscitation, 75, 491-498. https://doi.org/10.1016/j.resuscitation.2007.05.014

[17] Shida, H., Matsuyama, T., Kiyohara, K., Kitamura, T., Kishimori, T., Kiguchi, T., et al. (2019) Prehospital Cardiopulmonary Resuscitation Duration and Neurological Outcome after Out-of-Hospital Cardiac Arrest among Children by Location of Arrest: A Nationwide Cohort Study. Scandinavian Journal of Trauma, Resuscitation and Emergency Medicine, 27, 79. https://doi.org/10.1186/s13049-019-0658-7

[18] Thielen, M., Joshi, R., Delbressine, F., Bambang Oetomo, S. and Feijs, L. (2017) An Innovative Design for Cardiopulmonary Resuscitation Manikins Based on a $\mathrm{Hu}-$ man-Like Thorax and Embedded Flow Sensors. Proceedings of the Institution of Mechanical Engineers, Part H, 231, 243-249. https://doi.org/10.1177/0954411917691555

[19] Erdinc, R. (2019) Mecca, Saudi Arabia, Hosts First Global Project for "Heart Safe City".

https://www.philips.sa/en/a-w/about/news/archive/standard/news/press/2019/2019 0129-mecca-saudi-arabia-hosts-first-global-project-for-heart-safe-city.html

[20] Nehme, Z., Namachivayam, S., Forrest, A., Butt, W., Bernard, S. and Smith, K. (2018) Trends in the Incidence and Outcome of Paediatric Out-of-Hospital Cardiac Arrest: A 17-Year Observational Study. Resuscitation, 128, 43-50. https://doi.org/10.1016/j.resuscitation.2018.04.030

[21] ZOLL Medical Corporation. LifeVest Wearable Defibrillator. http://lifevest.zoll.com

[22] Wissenberg, M., Lippert, F.K., Folke, F., Weeke, P., Hansen, C.M., Christensen, E.F., Jans, H., Hansen, P.A., Lang-Jensen, T., Olesen, J.B. and Lindhardsen, J. (2013) Association of National Initiatives to Improve Cardiac Arrest Management with Rates of Bystander Intervention and Patient Survival after Out-of-Hospital Cardiac Arrest. JAMA, 10, 1377-1384. https://doi.org/10.1001/jama.2013.278483

[23] Berdowski, J., Berg, R.A., Tijssen, J.G. and Koster, R.W. (2010) Global Incidences of Out-of-Hospital Cardiac Arrest and Survival Rates: Systematic Review of 67 Prospective Studies. Resuscitation, 81, 1479-1487. https://doi.org/10.1016/j.resuscitation.2010.08.006

[24] Li, Q., Zhou, R.H., Liu, J., Lin, J., Ma, E.L., Liang, P., Shi, T.W., Fang, L.Q. and Xiao, H. (2013) Pre-Training Evaluation and Feedback Improved Skills Retention of Basic Life Support in Medical Students. Resuscitation, 84, 1274-1278. https://doi.org/10.1016/j.resuscitation.2013.04.017

[25] American College of Emergency Physicians (2006) Public Training in Cardiopulmonary Resuscitation and Public Access Defibrillation. Annals of Emergency Medicine, 47, 585. https://doi.org/10.1016/j.annemergmed.2006.02.024

[26] Hung, M.S., Lui, J.C., Lee, D.T., Shiu, I.Y. and Choi, K.C. (2014) Public Knowledge and Attitudes towards Cardiopulmonary Resuscitation in Hong Kong: Telephone Survey. Hong Kong Medical Journal, 20, 126-133.

[27] Fernandes, J.M., Leite, A.L., Auto, B.D., Lima, J.E., Rivera, I.R. and Mendonça, M.A. (2014) Teaching Basic Life Support to Students of Public and Private High Schools. Arquivos Brasileiros de Cardiologia, 102, 593-601. https://doi.org/10.5935/abc.20140071

[28] Fanning, R.M. and Gaba, D.M. (2007) The Role of Debriefing in Simulation-Based Learning. SIM Healthcare, 2, 115-125. 
https://doi.org/10.1097/SIH.0b013e3180315539

[29] Riegel, B., Nafziger, S.D., McBurnie, M.A., Powell, J., Ledingham, R., Selvia, R., et al. (2006) How Well Are Cardiopulmonary Resuscitation and Automated External Defibrillator Skills Retained over Time? Results from the Public Access Defibrillation (PAD) Trial. Academic Emergency Medicine, 13, 254-263. https://doi.org/10.1197/j.aem.2005.10.010

[30] Thiagarajan, S. (1992) Using Games for Debriefing. SIM Gaming, 23, 161-173. https://doi.org/10.1177/1046878192232004

[31] Hazinski, M.F., Nolan, J.P., Billi, J.E., Böttiger, B.W., Bossaert, L., de Caen, A.R., et al. (2010) Part 1: Executive Summary: 2010 International Consensus on Cardiopulmonary Resuscitation and Emergency Cardiovascular Care Science with Treatment Recommendations. Circulation, 122, S250-S275. https://doi.org/10.1161/CIRCULATIONAHA.110.970897

[32] Woollard, M., Whitfiled, R., Smith, A., et al. (2004) Skill Acquisition and Retention in Automated External Defibrillator (AED) Use and CPR by Lay Responders: A Prospective Study. Resuscitation, 60, 17-28. https://doi.org/10.1016/j.resuscitation.2003.09.006

[33] Edelson, D.P., Litzinger, B., Arora, V., Walsh, D., Kim, S., Lauderdale, D.S., et al. (2008) Improving In-Hospital Cardiac Arrest Process and Outcomes with Performance Debriefing. Archives of Internal Medicine, 168, 1063-1069. https://doi.org/10.1001/archinte.168.10.1063

[34] Korttila, K., Vertio, H. and Savolainen, K. (1979) Importance of Using Proper Techniques to Teach Cardiopulmonary Resuscitation to Laymen. Acta Anaesthesiologica Scandinavica, 23, 235-241. https://doi.org/10.1111/j.1399-6576.1979.tb01445.x

[35] Ribeiro, L.G., Germano, R., Menezes, P.L., Schmidt, A. and Pazin-Filho, A. (2013) Medical Students Teaching Cardiopulmonary Resuscitation to Middle School Brazilian Students. Arquivos Brasileiros de Cardiologia, 101, 328-335. https://doi.org/10.5935/abc.20130165

[36] Finn, J.C., Bhanji, F., Lockey, A., Monsieurs, K., Frengley, R., Iwami, T., et al. (2015) Part 8: Education, Implementation, and Teams: 2015 International Consensus on Cardiopulmonary Resuscitation and Emergency Cardiovascular Care Science with Treatment Recommendations. Resuscitation, 95, e203-e224.

[37] Sigsbee, M. and Geden, E.A. (1990) Effects of Anxiety on Family Members of Patients with Cardiac Disease Learning Cardiopulmonary Resuscitation. Heart \& Lung, 19, 662-665.

[38] Miotto, H.C., Camargos, F.R.D.S., Ribeiro, C.V., Goulart, E. and Moreira, M.D.C.V. (2010) Effects of the Use of Theoretical versus Theoretical-Practical Training on Cardiopulmonary Resuscitation. Arquivos Brasileiros de Cardiologia, 95, 328-331. https://doi.org/10.1590/S0066-782X2010005000104

[39] Everett-Thomas, R., Turnbull-Horton, V., Valdes, B., Valdes, G.R., Rosen, L.F. and Birnbach, D.J. (2016) The Influence of High Fidelity Simulation on First Responders, Retention of CPR Knowledge. Applied Nursing Research, 30, 94-97. https://doi.org/10.1016/j.apnr.2015.11.005

[40] Papalexopoulou, K., Chalkias, A., Dontas, I., Pliatsika, P., Giannakakos, C., Papapanagiotou, P., et al. (2014) Education and Age Affect Skill Acquisition and Retention in Lay Rescuers after a European Resuscitation Council CPR/AED Course. Heart \& Lung, 43, 66-71. https://doi.org/10.1016/j.hrtlng.2013.09.008 